\title{
Toward multimodality oral cancer diagnosis in the XXI century: Blending cutting edge imaging and genomic/proteomic definition of suspicious lesions
}

\author{
Anh Le ${ }^{1}$, Diana Messadi ${ }^{2}$, Joel Epstein ${ }^{3}$, Petra Wilder-Smith ${ }^{4}$
}

${ }^{1}$ Center for Craniofacial Molecular Biology, University of Southern California, School of Dentistry, Los Angeles, CA 90033; ${ }^{2}$ Section of Oral Medicine, Division of Oral Biology \& Medicine, UCLA School of Dentistry, University of California, Los Angeles, CA 90095; ${ }^{3}$ College of Dentistry and Cancer Center, College of Medicine, University of Illinois at Chicago, Chicago, IL 60612; ${ }^{4}$ Beckman Laser Institute, University of California, Irvine 1002 Health Sciences Rd East, Irvine, CA 92612; Petra Wilder-Smith - Email: pwsmith@uci.edu ; *Corresponding author

Received September 17, 2010; Accepted October 9, 2010; Published January 06, 2011

Abstract:

If emergent genomic and proteomic approaches to early oral cancer detection are to be successful, a means of reliably and comprehensively identifying high-risk tissue sampling sites constitutes an essential step in the oral cancer screening process. Recent studies have determined that in vivo Optical Coherence Tomography (OCT) is a quick and user-friendly tool for detecting and mapping oral lesions, and that it can enhance diagnostic accuracy when using high resolution diagnostic techniques such as in vivo microscopy. Therefore OCT can potentially provide a means of improving the clinical usefulness of novel diagnostic approaches such as proteomics by identifying sites that need to be sampled

\section{Background:}

The development of genomic and proteomic techniques is widely envisioned as a useful and powerful approach for oral cancer biomarker discovery. However, if proteomic approaches are to be successful, a reliable and comprehensive means of identifying appropriate tissue sampling sites is essential in order to limit costly and time-consuming sampling to sites that truly may present a threat to the patient. The major focus of cancer proteomics is to identify target proteins for specific cancer therapy and protein biomarkers for early diagnosis and prognosis. The major drawback when looking for tumor specific biomarkers is the need to extract the tumor material either surgically or through a needle biopsy which is quite invasive, and needs specific clinical skills. Thus it is important that proteomic sampling be directed and limited to sites that truly are in need of sampling.

Squamous cell carcinoma, accounting for $96 \%$ of all oral cancers, is usually preceded by dysplasia presenting as white (leukoplakia), or occasionally red (erythroplakia) epithelial lesions on the oral mucosa. Leukoplakias develop in $1-4 \%$ of the population [1, 2]. Malignant transformation, which is quite unpredictable, occurs in $1-40 \%$ of leukoplakias over 5 years. Dysplastic lesions in the form of erythroplakias carry a risk for malignant conversion of $90 \%[1,2]$ with increasing severity of dysplasia associated with higher risk of progression to cancer. Since clinical examination with white light has been shown to vary with the experience level of the clinician and there remains no reliable correlation between clinical appearance and the presence of dysplastic changes detection, current practice prescribes frequent biopsy of suspicious oral lesions, to ensure early detection of malignant changes. However, biopsied lesions are many times benign and quite often may not represent the site of dysplastic changes, which, in combination with poor patient acceptance of surgical sampling, discomfort, and some degree of scarring, serves as a powerful disincentive to patients to attend for further regular surveillance.
Tumor detection is further complicated by a potential wide field (regional) molecular change with risk of progression to cancer, leading to multicentric lesions [4]. Especially in the case of tumor recurrence, and second primary tumors which are quite frequent in oral cancer, these lesions may not be visible to the naked eyes. Consequently, they are neither detected nor sampled and remain undiagnosed and untreated, posing a serious threat to a patient's long-term health. Thus, there exists an urgent need for a clinical modality for direct, non-invasive early detection and mapping of potentially (pre-) malignant oral lesions. We hypothesize that the combined use of a quick and noninvasive imaging approach to detect and map regions of interest would aid clinical examination and monitoring without warranting a biopsy specimen, as well as providing an indicator of the appropriate sites for proteomic characterization. In the head and neck region, early detection and monitoring of dysplastic and cancerous conditions will facilitate less aggressive treatment and result in a better prognosis.

\section{Method and Discussion:}

Optical Coherence Tomography (OCT) was first introduced as an imaging technique in biological systems in 1991 [3]. The non-invasive nature of this imaging modality coupled with (i) a penetration depth of 2-3 mm, (ii) high resolution $(1-10 \mu \mathrm{m})$, real-time image viewing, and (iii) capability for cross-sectional as well as 3-D tomographic images, provide excellent prerequisites for in vivo oral screening and diagnosis.

OCT has often been compared to ultrasound imaging. Both technologies employ backscattered signals reflected from different layers within the tissue to reconstruct structural images, with OCT measuring light rather than sound. The resulting OCT image is a two-dimensional representation of the optical reflection from a tissue sample. Cross-sectional images of tissues are constructed in real time, at near histologic resolution 


\section{Bioinformation}

(approximately 1-10 $\mu$ m with current technology). These images can be stacked to generate 3-D reconstructions of the target tissue. This permits in vivo non-invasive imaging of epithelial and subepithelial structures, including: (1) depth and thickness, (2) histopathological appearance, and (3) peripheral margins of the lesions.

Various OCT systems have received FDA approval for clinical use, and OCT is deemed by many as an essential imaging modality in ophthalmology. In vivo image acquisition is facilitated through the use of a flexible fiberoptic OCT probe. The probe is simply placed on the surface of the tissue to generate real-time, immediate surface and sub-surface images of tissue microanatomy and cellular structure, whilst avoiding the discomfort, delay and expense of biopsies.

Several studies have sought to investigate the diagnostic utility of in vivo OCT to detect and diagnose oral pre-malignancy and malignancy $[\mathbf{4}, \mathbf{5}]$. In a blinded study involving 50 patients with clinically suspicious lesions including oral leukoplakia or erythroplakia the effectiveness of OCT was evaluated for detecting oral dysplasia and malignancy [5]. OCT images of dysplastic lesions revealed visible epithelial thickening, loss of epithelial stratification, and epithelial down-growth (Figure 1). Areas of ora squamous cell carcinoma of the buccal mucosa were identified in the OCT images by the absence or disruption of the basement membrane, an epithelial layer that was highly variable in thickness, with areas of erosion and extensive epithelial down-growth and invasion into the sub-epithelial layers (Figure 2). A semi-quantified scale of 0-6 measuring these changes was developed and validated in animal and human studies [5, 8] Statistical analysis of the data gathered in this study using 50 subjects with oral lesions substantiated the utility of in vivo OCT to detect and diagnose oral pre-malignancy and malignancy in the oral cavity, with diagnostic accuracy, and early histological changes during clinical follow-up of the lesions. Agreement between OCT and histopathology was excellent, averaging 0.896 ( $\mathrm{SE}=0.049)$. For detecting carcinoma in situ or squamous cell carcinoma (SCC) vs. non-cancer, sensitivity was 0.931 and specificity was 0.931; for detecting SCC vs. all other pathologies, sensitivity was 0.931 and specificity was 0.973 .

In another study of 97 patients using OCT imaging to detect neoplasia in the oral cavity [6], a sensitivity of $83 \%$ and specificity of $98 \%$ were determined with inter-observer agreement of 0.76 . This study suggested that OCT is a promising imaging modality for non-invasive detection, mapping, and evaluation of tissue sites suspicious for high-grade dysplasia or cancer. These findings also have important clinical diagnostic utility in detecting dysplastic and malignant lesions in patients previously treated for upper aerodigestive cancers, who are susceptible for new or recurrent cancer and who are difficult to assess clinically due to prior treatment induced mucosal change, and represent the highest risk patient population for OSCC. Therefore, several studies have demonstrated the potential role of OCT imaging for detecting, diagnosing and mapping oral lesions. In all of these studies, histopathology was used as the gold standard, which is indeed, the current accepted practice. Several other imaging approaches with promising advances in the last decade include photosensitizers, fluorescence, various forms of spectroscopy [7]. However, as we strive to define and validate more accurate markers of early cancer risk using genomic and proteomic approaches, the tissue sites to be interrogated by these markers - which, by their very definition are highly resolved and highly sensitive - must be identified with accuracy and precision. In an early animal study, we were able to determine that the diagnostic accuracy for oral malignancy of high-resolution in vivo microscopy was considerably enhanced by pre-scanning with OCT to identify and map oral lesions [8]. Hence it seems reasonable to propose that the use of OCT imaging techniques for identifying sites in need of genomic and proteomic interrogation will serve to enhance the accuracy, speed and comprehensiveness of the diagnostic process of early pre-malignant and malignant lesions in head and neck cancer.

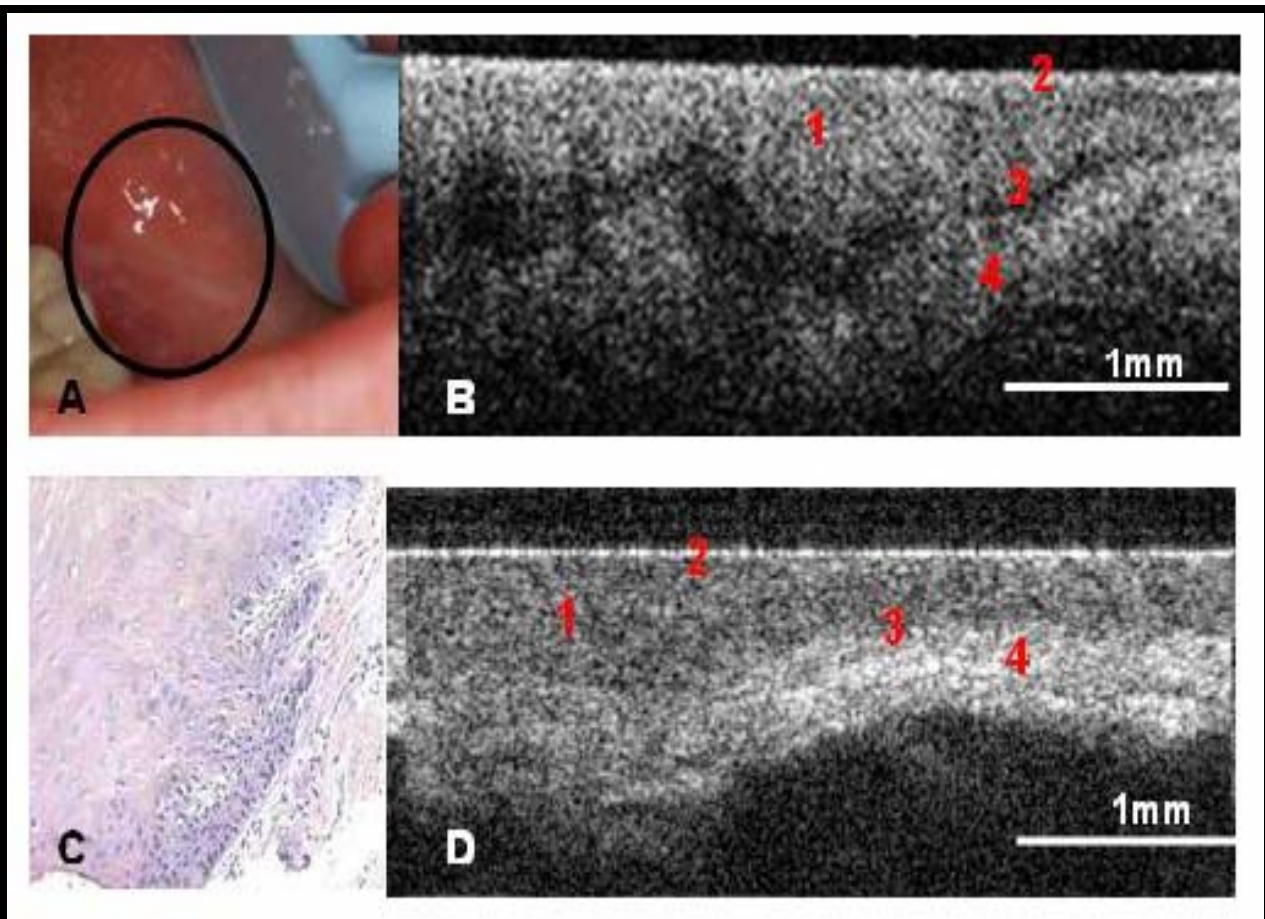

Figure 1: Dysplastic and Normal Buccal Mucosa. (A) Photograph, (B) in vivo Optical Coherence Tomography image, (C) H\&E (10x) of dysplastic buccal mucosa, (D) In vivo OCT image of normal buccal mucosa. Key: 1-stratified squamous epithelium, 2-keratinized epithelial surface layer, 3basement membrane 4-submucosa. From: "In-Vivo Diagnosis of Oral Dysplasia and Malignancy Using Optical Coherence Tomography: Preliminary Studies in 50 Patients. Wilder-Smith et al 2009. Reprinted with permission of Wiley-Liss, Inc. a subsidiary of John Wiley \& Sons, Inc.” 


\section{Bioinformation}
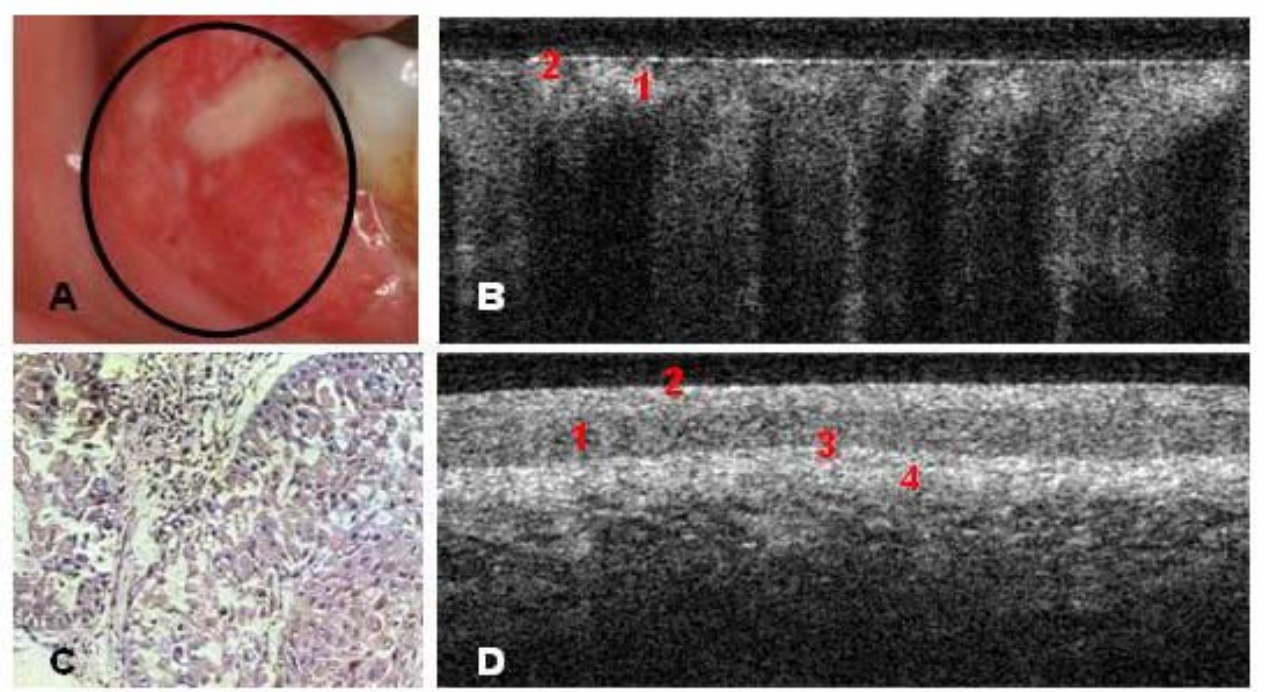

Figure 2: Squamous Cell Carcinoma of the Buccal Mucosa. (A) Photograph, (B) in vivo OCT image (C) H\&E (10x) of buccal mucosa with squamous cell carcinoma (D) In vivo OCT image of normal buccal mucosa. Key: 1-stratified squamous epithelium, 2-keratinized epithelial surface layer, 3-basement membrane 4-submucosa. From: "In-Vivo Diagnosis of Oral Dysplasia and Malignancy Using Optical Coherence Tomography: Preliminary Studies in 50 Patients. Wilder-Smith et al 2009. Reprinted with permission of Wiley-Liss, Inc. a subsidiary of John Wiley \& Sons, Inc.”

\section{Conclusion:}

In vivo imaging can identify and map oral premaligant and malignant lesions, providing a useful means for ensuring accurate and comprehensive sampling of oral lesions by emerging approaches such as genomics and proteomics. The increase in sensitivity and specificity of the combined optical imaging and proteomic technology when compared with both clinical examination and frozen section analysis supports their cutting edge approach for the XXI century of oral cancer detection and diagnosis.

\section{References:}

[1] American Cancer Society, Cancer facts and figures In: American Cancer Society Report, (2000) pp 1
[2] J Regezi \& J Sciubba, Eds. Oral pathology. Philadelphia: W.B. Saunders Co. (1993) pp 77

[3] D Huang et al. Science 254: 1178 (1991) [PMID: 1957169]

[4] MT Tsai et al. J Biomed Opt. 13: 44012 (2008)

[5] P Wilder-Smith et al. Lasers Surg Med. 41: 353 (2009) [PMID: 19533765]

[6] MT Tsai et al. Opt Express. 16: 15847 (2008)

[7] M DeCoro \& P Wilder-Smith Expert, Rev. Anticancer Ther. 10: 321 (2010) [PMID: 20214513]

[8] P Wilder-Smith et al. J. Biomed. Opt. 10: 051601 (2005) [PMID:16292949]

Edited by F Chiappelli

Citation: Le et al. Bioinformation 5(7): 304-306 (2011) License statement: This is an open-access article, which permits unrestricted use, distribution, and reproduction in any medium, for non-commercial purposes, provided the original author and source are credited. 